\title{
Flexible transbronchial optical frequency domain imaging smart needle for biopsy guidance
}

\section{Citation}

Tan, K. M., M. Shishkov, A. Chee, M. B. Applegate, B. E. Bouma, and M. J. Suter. 2012. Flexible transbronchial optical frequency domain imaging smart needle for biopsy guidance. Biomedical Optics Express 3(8): 1947-1954.

\section{Published Version}

doi:10.1364/BOE.3.001947

\section{Permanent link}

http://nrs.harvard.edu/urn-3:HUL.InstRepos:10465994

\section{Terms of Use}

This article was downloaded from Harvard University's DASH repository, and is made available under the terms and conditions applicable to Other Posted Material, as set forth at http:// nrs.harvard.edu/urn-3:HUL.InstRepos:dash.current.terms-of-use\#LAA

\section{Share Your Story}

The Harvard community has made this article openly available.

Please share how this access benefits you. Submit a story.

\section{Accessibility}




\title{
Flexible transbronchial optical frequency domain imaging smart needle for biopsy guidance
}

\author{
K. M. Tan, ${ }^{1,2,3}$ M. Shishkov, ${ }^{2,3}$ A. Cheee,${ }^{1,2,3}$ M. B. Applegate,${ }^{1,2}$ B. E. Bouma,,${ }^{2,3,4,5}$ and \\ M. J. Suter ${ }^{1,2,3,4, *}$ \\ ${ }^{I}$ Department of Medicine, Pulmonary and Critical Care Unit, Massachusetts General Hospital, \\ 40 Blossom Street, Boston, Massachusetts, 02114, USA \\ ${ }^{2}$ Wellman Center for Photomedicine, Massachusetts General Hospital, 50 Blossom Street, Boston, \\ Massachusetts, 02114, USA \\ ${ }^{3}$ Harvard Medical School, 25 Shattuck Street, Boston, Massachusetts, 02115, USA \\ ${ }^{4}$ Equal contribution \\ 5bbouma@partners.org \\ *msuter@partners.org
}

\begin{abstract}
Transbronchial needle aspiration (TBNA) is a procedure routinely performed to diagnose peripheral pulmonary lesions. However, TBNA is associated with a low diagnostic yield due to inappropriate needle placement. We have developed a flexible transbronchial optical frequency domain imaging (TB-OFDI) catheter that functions as a "smart needle" to confirm the needle placement within the target lesion prior to biopsy. The TB-OFDI smart needle consists of a flexible and removable OFDI catheter (430 $\mu \mathrm{m}$ dia.) that operates within a standard 21-gauge TBNA needle. The OFDI imaging core is based on an angle polished ball lens design with a working distance of $160 \mu \mathrm{m}$ from the catheter sheath and a spot size of $25 \mu \mathrm{m}$. To demonstrate the potential of the TB-OFDI smart needle for transbronchial imaging, an inflated excised swine lung was imaged through a standard bronchoscope. Cross-sectional and longitudinal OFDI results reveal the detailed network of alveoli in the lung parenchyma suggesting that the TB-OFDI smart needle may be a useful tool for guiding biopsy acquisition to increase the diagnostic yield.
\end{abstract}

(C) 2012 Optical Society of America

OCIS codes: (170.4500) Optical coherence tomography; (170.4580) Optical diagnostics for medicine; (170.3890) Medical optics instrumentation; (170.3880) Medical and biological imaging; (170.2150) Endoscopic imaging; (060.2350) Fiber optics imaging.

\section{References and links}

1. America Cancer Society, "Cancer facts and figures 2010" (ACS, 2010).

2. S. Altekruse, C. Kosary, M. Krapcho, N. Neyman, R. Aminou, W. Waldron, J. Ruhl, N. Howlader, Z Tatalovich, and H. Cho, "SEER cancer statistics review" (National Cancer Institute, Bethesda, MD, 2010).

3. A. B. Mariotto, K. R. Yabroff, Y. Shao, E. J. Feuer, and M. L. Brown, "Projections of the cost of cancer care in the United States: 2010-2020," J. Natl. Cancer Inst. 103(2), 117-128 (2011).

4. W. A. Baaklini, M. A. Reinoso, A. B. Gorin, A. Sharafkaneh, and P. Manian, "Diagnostic yield of fiberoptic bronchoscopy in evaluating solitary pulmonary nodules," Chest 117(4), 1049-1054 (2000).

5. D. Huang, E. A. Swanson, C. P. Lin, J. S. Schuman, W. G. Stinson, W. Chang, M. R. Hee, T. Flotte, K. Gregory, C. A. Puliafito, and et, "Optical coherence tomography," Science 254(5035), 1178-1181 (1991).

6. S. Yun, G. Tearney, J. de Boer, N. Iftimia, and B. Bouma, "High-speed optical frequency-domain imaging," Opt. Express 11(22), 2953-2963 (2003).

7. M. Tsuboi, A. Hayashi, N. Ikeda, H. Honda, Y. Kato, S. Ichinose, and H. Kato, "Optical coherence tomography in the diagnosis of bronchial lesions," Lung Cancer 49(3), 387-394 (2005).

8. S. Lam, B. Standish, C. Baldwin, A. McWilliams, J. leRiche, A. Gazdar, A. I. Vitkin, V. Yang, N. Ikeda, and C. MacAulay, "In vivo optical coherence tomography imaging of preinvasive bronchial lesions," Clin. Cancer Res. 14(7), 2006-2011 (2008).

9. R. G. Michel, G. T. Kinasewitz, K. M. Fung, and J. I. Keddissi, “Optical coherence tomography as an adjunct to flexible bronchoscopy in the diagnosis of lung cancer: a pilot study," Chest 138(4), 984-988 (2010). 
10. H. O. Coxson, B. Quiney, D. D. Sin, L. Xing, A. M. McWilliams, J. R. Mayo, and S. Lam, "Airway wall thickness assessed using computed tomography and optical coherence tomography," Am. J. Respir. Crit. Care Med. 177(11), 1201-1206 (2008).

11. J. J. Armstrong, M. S. Leigh, D. D. Sampson, J. H. Walsh, D. R. Hillman, and P. R. Eastwood, "Quantitative upper airway imaging with anatomic optical coherence tomography," Am. J. Respir. Crit. Care Med. 173(2), 226-233 (2006).

12. X. D. Li, C. Chudoba, T. Ko, C. Pitris, and J. G. Fujimoto, "Imaging needle for optical coherence tomography," Opt. Lett. 25(20), 1520-1522 (2000).

13. V. X. D. Yang, Y. X. Mao, N. Munce, B. Standish, W. Kucharczyk, N. E. Marcon, B. C. Wilson, and I. A. Vitkin, "Interstitial Doppler optical coherence tomography," Opt. Lett. 30(14), 1791-1793 (2005).

14. A. M. Zysk, D. L. Marks, D. Y. Liu, and S. A. Boppart, "Needle-based reflection refractometry of scattering samples using coherence-gated detection," Opt. Express 15(8), 4787-4794 (2007).

15. D. Lorenser, X. Yang, R. W. Kirk, B. C. Quirk, R. A. McLaughlin, and D. D. Sampson, "Ultrathin sideviewing needle probe for optical coherence tomography," Opt. Lett. 36(19), 3894-3896 (2011).

16. Y Wu, J. Xi, L. Huo, J. Padvorac, E. J. Shin, S. A. Giday, A. A. Lennon, M. I. F. Canto, J. H. Hwang, and X. Li, "Robust high-resolution fine OCT needle for side-viewing interstitial tissue imaging," IEEE J. Sel. Top. Quantum Electron. 16(4), 863-869 (2010).

17. B. C. Quirk, R. A. McLaughlin, A. Curatolo, R. W. Kirk, P. B. Noble, and D. D. Sampson, "In situ imaging of lung alveoli with an optical coherence tomography needle probe,” J. Biomed. Opt. 16(3), 036009 (2011).

18. W. C. Kuo, J. Kim, N. D. Shemonski, E. J. Chaney, D. R. Spillman, Jr., and S. A. Boppart, "Real-time three-dimensional optical coherence tomography image-guided core-needle biopsy system," Biomed. Opt. Express 3(6), 1149-1161 (2012).

19. M. J. Suter, B. J. Vakoc, P. S. Yachimski, M. Shishkov, G. Y. Lauwers, M. Mino-Kenudson, B. E. Bouma, N. S. Nishioka, and G. J. Tearney, "Comprehensive microscopy of the esophagus in human patients with optical frequency domain imaging," Gastrointest. Endosc. 68(4), 745-753 (2008).

20. S. H. Yun, G. J. Tearney, J. F. de Boer, N. Iftimia, and B. E. Bouma, "High-speed optical frequency-domain imaging," Opt. Express 11(22), 2953-2963 (2003).

21. J. H. Han, X. Liu, C. G. Song, and J. U. Kang, "Common path optical coherence tomography with fibre bundle probe," Electron. Lett. 45(22), 1110-1112 (2009).

22. S. Han, M. V. Sarunic, J. Wu, M. Humayun, and C. H. Yang, "Handheld forward-imaging needle endoscope for ophthalmic optical coherence tomography inspection," J. Biomed. Opt. 13(2), 020505 (2008).

23. C. P. Liang, J. Wierwille, T. Moreira, G. Schwartzbauer, M. S. Jafri, C. M. Tang, and Y. Chen, "A forwardimaging needle-type OCT probe for image guided stereotactic procedures," Opt. Express 19(27), 2628326294 (2011).

24. E. J. Min, J. Na, S. Y. Ryu, and B. H. Lee, "Single-body lensed-fiber scanning probe actuated by magnetic force for optical imaging," Opt. Lett. 34(12), 1897-1899 (2009).

\section{Introduction}

Lung cancer is the leading cause of cancer related death [1-3]. It is well established that early detection and diagnosis greatly increases patient survival [4]. Macroscopic imaging techniques such as computed tomography (CT) are highly sensitive at detecting small, $\leq$ $2 \mathrm{~cm}$, peripheral pulmonary lesions (PPLs) in the lung but they lack the specificity necessary for diagnosis. Diagnosis of malignancy must therefore be made by excisional biopsy, which may be accomplished by high-risk surgical approaches, percutaneous biopsy, or with low-risk approaches such as bronchoscopy with transbronchial needle aspiration (TBNA). Both endobronchial ultrasound (EBUS) guided and unguided TBNA are associated with unacceptably low diagnostic yields for small lesions and therefore higher-risk procedures are often preferentially performed [4]. We anticipate that a smart TBNA needle that provides confirmation of the needle placement within the lesion of interest prior to biopsy will result in an increase in the diagnostic yield.

Optical frequency domain imaging (OFDI) is a high-speed second-generation optical coherence tomography (OCT) imaging modality that can be used to generate real-time cross-sectional images of tissue microstructure to depths approaching $3 \mathrm{~mm}[5,6]$. Studies have shown that OCT may be a useful tool for differentiating preinvasive cancers of the bronchial mucosa [7-9], and may provide a useful method for assessing distal airway wall remodeling [10] as well as providing quantitative measurements of upper airway size and shape [11]. Due to limitations in catheter designs, OCT/OFDI has been traditionally restricted to imaging of luminal organs, however the development of needle-based catheters promises to extend the utility of OCT beyond these boundaries. For minimally invasive interstitial imaging of tissues and organs, small diameter rigid OCT needle probes have been developed [12-17]. In these designs the imaging optics, which typically consist of a ball-lens [13] or a fiber gradient-index (GRIN) lens [15-17] design, are housed within the stainless steel hypodermic needles. To enable circumferential scanning, 
a notch is cut in one side of the needle to form a window, and the entire needle is rotated through $360^{\circ}$. Limitations to the design of these OCT needles include the inability to obtain tissue specimens for diagnosis through the same imaging needle, the incompatibility with standard endoscopy procedures, and due to the direct contact of the mechanically scanning needle with the tissue, unintentional tissue damage or tissue drag may occur distorting the images acquired. Recently, Wei-Cheng Kuo et al. demonstrated an OCT-guided core-needle biopsy system incorporating a vacuum-assisted rigid breast needle biopsy console, which was modified to accommodate the OCT imaging probe [18]. This work highlights the potential of OCT image guidance for biopsy site selection, but at present is limited to rigid needle designs and therefore is not compatible with transbronchial procedures.

We have developed a narrow diameter $(0.43 \mathrm{~mm})$ flexible helical scanning OFDI imaging catheter that is compatible with standard 21-guage bronchoscopy TBNA needles (Olympus NA-2001SX). The helically scanning optical fiber imaging core is housed within a stationary $430 \mu \mathrm{m}$ outer-diameter polyimide catheter sheath, and the entire flexible catheter operates within the TBNA needle to form the complete TB-OFDI smart needle. During imaging, only the optical fiber imaging core rotates while the catheter sheath remains stationary, thus reducing the risk of tissue damage. The TB-OFDI smart needle is designed to enable both OFDI imaging and subsequent specimen collection for diagnosis without removing or repositioning the TBNA needle. To test the usability and image quality of the TB-OFDI smart needle, we performed transbronchial imaging of an excised inflated swine lung. The lungs were inflated with air rather than saline to closely mimic in vivo conditions. Images of alveoli within the lung parenchyma were clearly visible.

\section{Materials and methods}

\subsection{Flexible OFDI imaging catheter}

Figure 1(a) illustrates the schematic diagram for the distal-end of the TB-OFDI smart needle. The OFDI catheter insert consists of the imaging core housed inside a transparent polyimide sheath. The imaging core consists of an optical fiber imaging probe and a nitinol drive shaft. The catheter slides freely in the TBNA needle.

Figure 1(b) shows a photograph of the side-polished ball-lens optics. The ball-lens was fabricated directly on the end of a $500 \mu \mathrm{m}$ long coreless fiber via tungsten filament splicer (Vytran FFS-2000). The proximal end of the coreless fiber was spliced to a single mode fiber (SMF28). The curvature of the ball-lens, which affects the focal point and beam spot size, was controlled by the length of the melted coreless fiber. The ball-lens was designed to have a focal length of $\sim 400 \mu \mathrm{m}$ to ensure positioning of the focal plane outside of the catheter sheath and in the surrounding tissue. The designed sagittal and tangential radii of the ball-lens were $75 \mu \mathrm{m}$ and $100 \mu \mathrm{m}$ respectively.

The ball-lens was polished at an angle of $36^{\circ}$ resulting in total internal reflection of the incident beam necessary for the side-viewing catheter design. Rather than a 90 degree emission angle, the beam is emitted at an $81^{\circ}$ forward viewing angle to reduce specular reflection that may arise from the catheter sheath and tissue interfaces. A $1.2 \mathrm{~m}$ long hollow nitinol driveshaft, with an outer diameter of $220 \mu \mathrm{m}$, was used to house and protect the imaging optics and to transmit the necessary mechanical torque for helical scanning. The entire optical core was then inserted into a catheter sheath. The sheath comprised two segments: over the majority of its length, the sheath material was polyimide with a polytetrafluoroethylene (PTFE) liner $(393.7 \mu \mathrm{m}$ outer diameter and 320 $\mu \mathrm{m}$ inner diameter) to minimize friction; the distal $7 \mathrm{~cm}$ portion of the sheath was polyimide alone $(430 \mu \mathrm{m}$ outer diameter and $380 \mu \mathrm{m}$ inner diameter) to optimize optical quality within the imaging segment (Fig. 1(c)). The two sheath portions were bonded together and the distal tip was sealed using 353NDT epoxy (Epotek) to both protect the patient from the mechanical scanning of the inner core and to ensure that the imaging optics do not get contaminated with bodily fluids. The proximal end of the imaging catheter was fitted with a custom rapid connect device to interface with the optical rotary junction and pullback tray of our existing clinical OFDI systems for volumetric imaging 

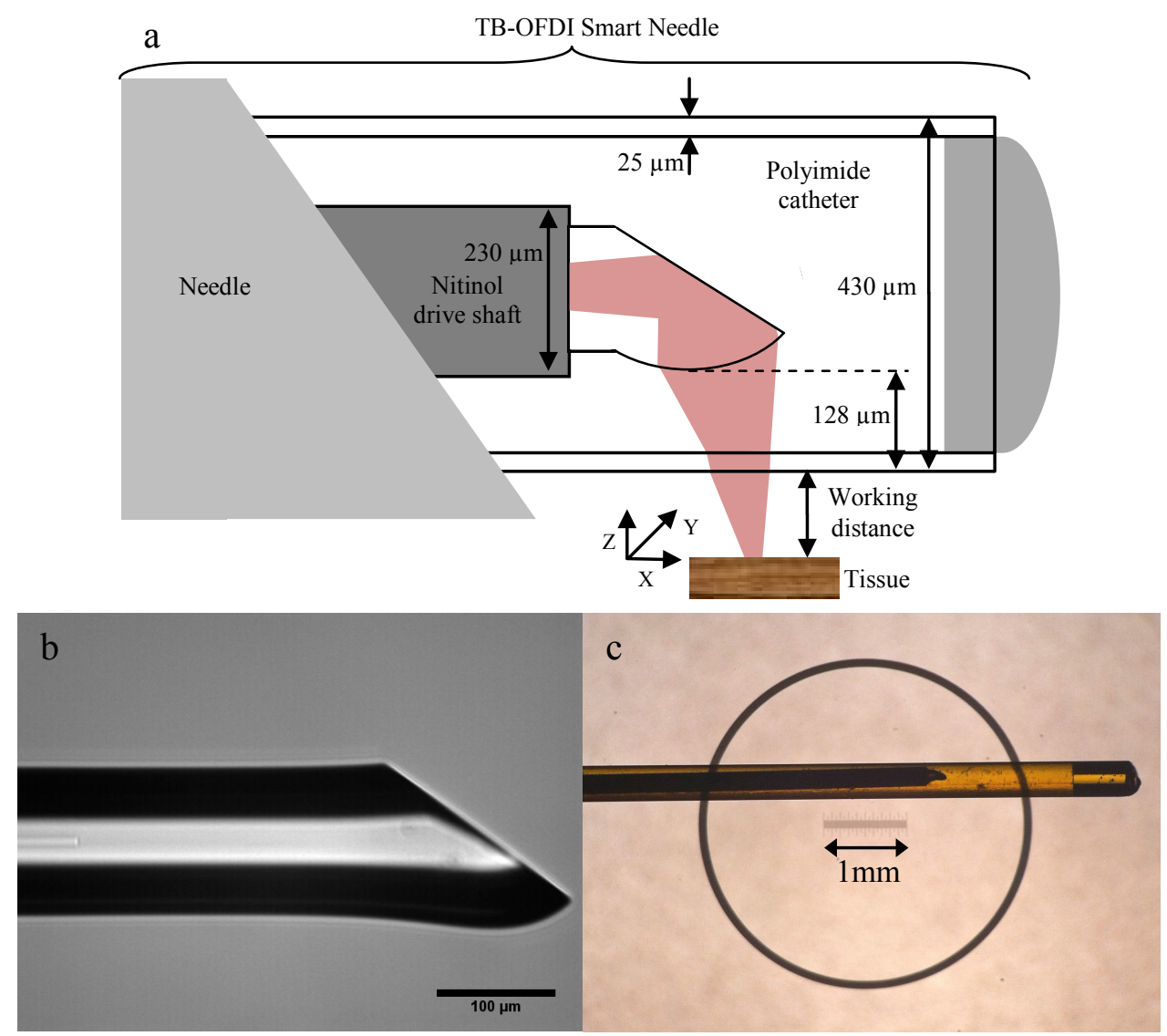

Fig. 1. (a) Schematic diagram of the TB-OFDI distal end. (b) Photograph of the polished ball-lens optical core. (c) Photograph of the flexible OFDI catheter insert. The inner optical core, which is encased in a nitinol driveshaft, is located within the sealed, optically transparent polyimide sheath.

[19]. Stiction will not be an issue, as the catheter sheath is encased within the needle, which provides the necessary external support.

\subsection{Modification of TBNA needle}

The OFDI catheter was designed to attach, via a luer lock fitting, to the proximal end of the TBNA needle (Olympus NA-201SX-4021) for imaging, Fig. 2(a). The catheter can be easily removed to facilitate tissue sampling. The TBNA needle used in this study was

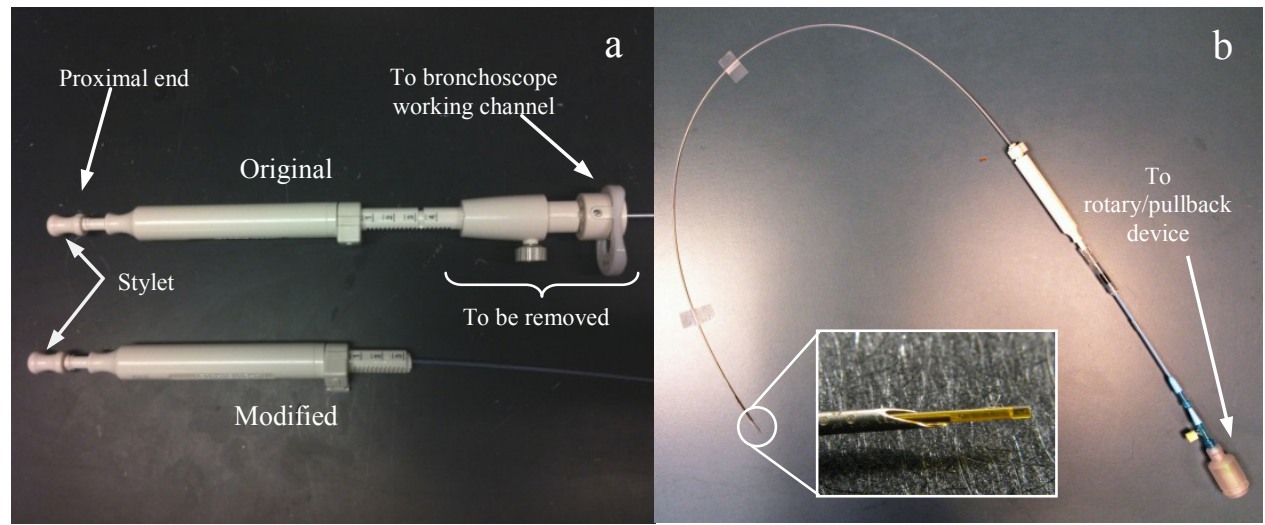

Fig. 2. (a) Original TBNA needle (top) and the modified TBNA needle (bottom) with a portion of the proximal assembly removed to increase the workable length. (b) Complete TB-OFDI smart needle with the OFDI catheter residing within the 21-gauge TBNA needle (inset). 
primarily designed for central lesions and was therefore not long enough to extend to the very peripheral regions of the lung. To extend the reachability of the TBNA needle we removed a section of the handle that interfaces with the bronchoscope. Removal of this piece did not compromise the functionality of the TBNA needle. Figure 2(b) shows a photograph of the complete TB-ODFI smart needle.

\subsection{OFDI system}

The technological approach to OFDI has been described in detail previously [20]. Briefly, the system used in this study consisted of a $40 \mathrm{kHz}$ wavelength swept source centered at $1320 \mathrm{~nm}$ with a FWHM sweep range of $143 \mathrm{~nm}$ [19]. The axial resolution in OFDI is determined by the range over which the wavelength is scanned [20], and in the current system resulted in an axial resolution of approximately $6 \mu \mathrm{m}$ in air (theoretical axial resolution is $5 \mu \mathrm{m}$ ). Data was acquired and continuously stored in a RAID high-speed data storage device at a rate of $85 \mathrm{MS} / \mathrm{sec}$ corresponding to a frame rate 9.8 frames/sec (frame size: 2048 x 4096). To facilitate volumetric imaging, the TB-OFDI catheter was connected to an optical rotary junction and pullback device in the sample arm.

\subsection{TB-OFDI imaging procedure}

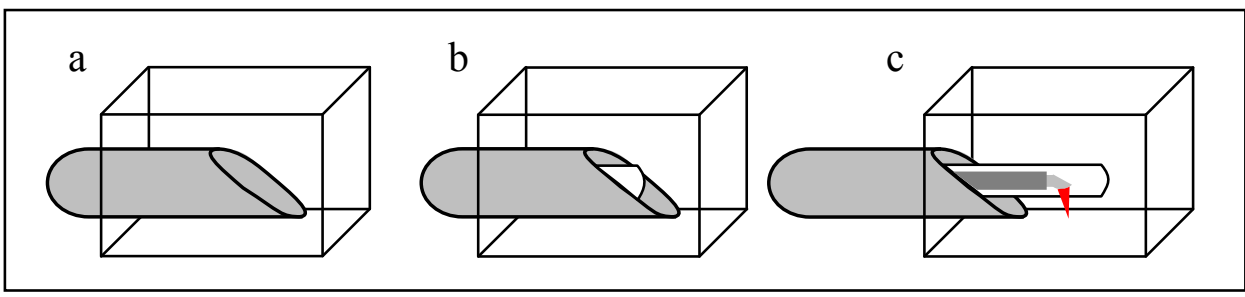

Fig. 3. Schematic demonstrates the functionality of the TB-OFDI smart needle. (a) The TBNA needle is placed in the lung tissue. (b) The OFDI catheter is advanced within the needle. (c) The TBNA needle is withdrawn exposing the OFDI catheter for imaging.

To demonstrate the feasibility and image quality of the TB-OFDI smart needle we imaged freshly excised lungs from swine. An endotracheal tube was inserted and inflated in the trachea and the lungs were subsequently inflated to a pressure of $20 \mathrm{cmH}_{2} \mathrm{O}$. A bronchoscope (1970K, Pentax, Japan) was then inserted into the endotracheal tube and the airways were examined. Following identification of a target site, the TBNA needle was inserted into the working channel of the bronchoscope and was maneuvered to puncture the airway wall and enter the parenchyma, Fig. 3(a). The TBNA stylet was subsequently withdrawn and was replaced with the OFDI catheter, Fig. 3(b). The OFDI catheter was locked onto the proximal end of the TBNA needle via a luer lock. Once the catheter was advanced to the distal end of the needle, the TBNA needle was withdrawn $\sim 1-2 \mathrm{~cm}$ to expose the OFDI catheter for imaging, Fig. 3(c). Following imaging with the OFDI catheter still in place, the TBNA needle was re-advanced over the catheter to the initial position, the OFDI catheter was then unlocked and removed from the TBNA needle, and an aspirate or core biopsy obtained for diagnosis. This procedure ensures that the TBNA needle remains within the target tissue for biopsy acquisition following OFDI imaging.

\section{Results}

\subsection{Optical properties}

As was previously mentioned, the purpose of the catheter sheath is to protect the patient from the mechanical scanning of the inner core and to ensure that the imaging optics do not get contaminated with bodily fluids. The downside, however, is the induced astigmatism by the encasing cylindrical shaped sheath. In an OCT needle developed by Wu et al., the authors reduced the astigmatism of their 25 -guage probe by filling the inner portion of the needle with index matching fluid and by polishing the outer cylindrical glass surface flat [16]. This solution nicely eliminated the astigmatism of the OCT beam at the focal position in the authors' rigid needle probe design, however this solution is not feasible in our flexible catheter design due to the presence of the protective sheath. Rather 

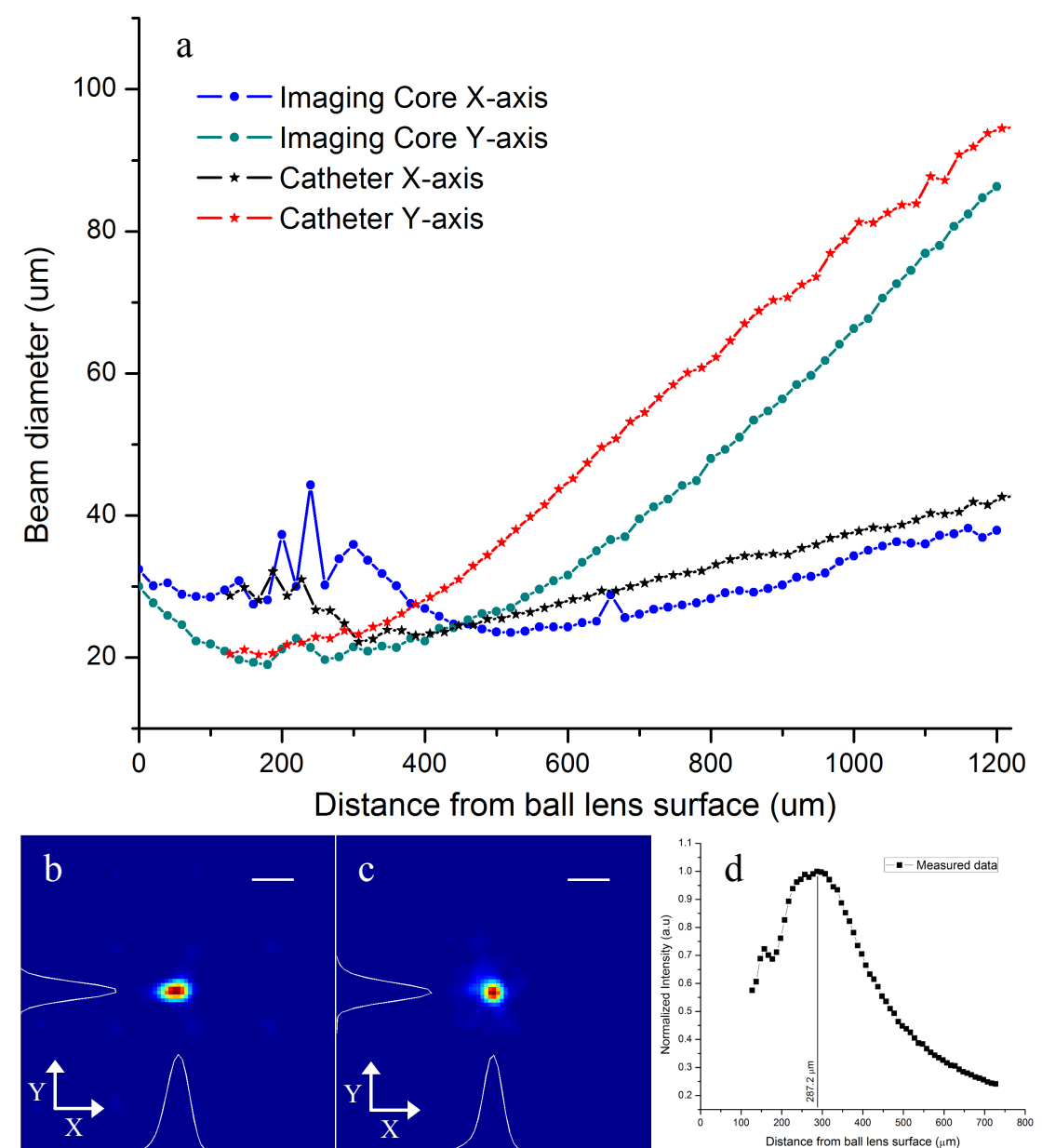

Fig. 4. (a) Output beam diameter of the imaging core (blue and dark cyan) and catheter (black and red). (b) and (c) Measured output beam profile of the imaging core, and the complete catheter respectively. (d) Measured beam intensity as a function of the distance from the surface of the ball lens. White scale bar indicates $50 \mu \mathrm{m}$.

than filling the inner portion of the sheath with index matching fluid to correct for the sheath-induced astigmatism, we designed our ball-lens to have an ellipsoid shape to compensate for the astigmatism. This design has an additional advantage in that the catheter sheath can be easily replaced between patients thus saving time and cost. To measure the effectiveness of astigmatism compensation, we used a CCD beam profiler (WinCamD, DataRay Inc.) to measure the beam profile of output beam of the imaging core and catheter. The catheter was mounted at an angle to ensure that the optical beam emitted from catheter was incident normal to the CCD chip.

Figure 4(a) shows the plots of experimentally measured beam diameter of both the imaging core and the catheter. The catheter beam diameter plot starts at $128 \mu \mathrm{m}$ which takes into account the air gap between the lens surface and the catheter sheath outer surface, see Fig. 1(a). The beam profile of the imaging core at the focal plane is elliptically shaped, as shown in Fig. 4(b). The measured $x$ - and y-axis beam diameters are $28 \mu \mathrm{m}$ and $19 \mu \mathrm{m}$, respectively. Using the ratio of $\mathrm{x}$-axis/y-axis, the astigmatism ratio is 1.47. The beam emitted from the catheter focuses $307 \mu \mathrm{m}$ from the lens surface or 179 $\mu \mathrm{m}$ from the outer edge of the catheter sheath. This value is in agreement with the measured working distance between the catheter sheath outer surface and the focal point, which is $\sim 160 \mu \mathrm{m}$ in Fig. 4(d). The beam profile, Fig. 4(c), of the complete OFDI catheter at the focus appears circular without significant astigmatism. The measured beam diameter in the $\mathrm{x}$ - and $\mathrm{y}$-axis is $23.9 \mu \mathrm{m}$ and $23 \mu \mathrm{m}$ respectively. This yields an 
astigmatism ratio reduction from 1.4 to 1.04 . The transmission efficiency of the catheter was approximately $85 \%$.

\subsection{Imaging of ex vivo lung}

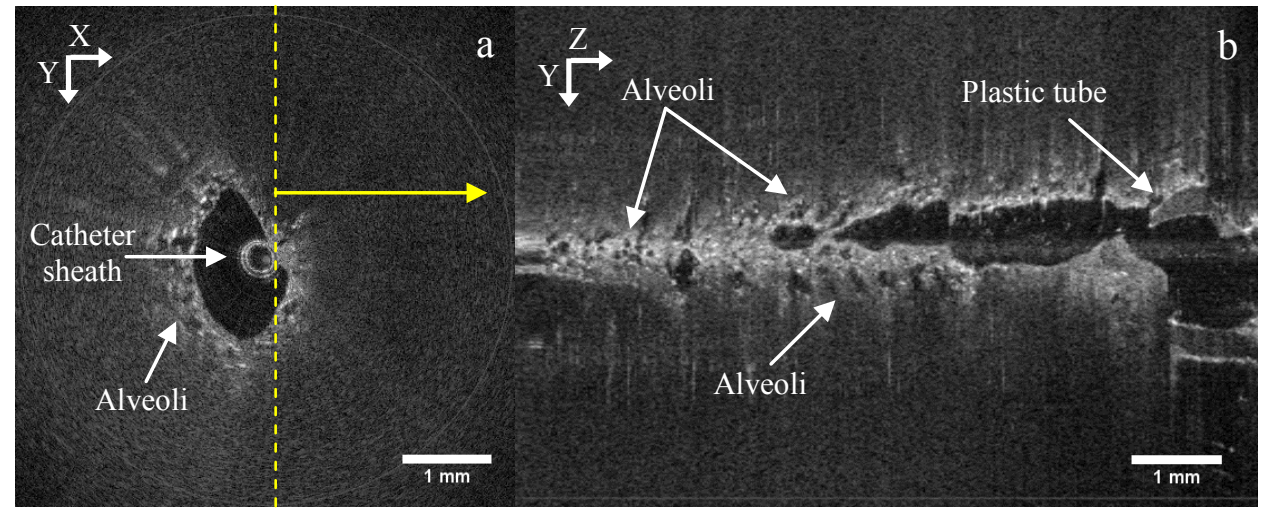

Fig. 5. Cross-sectional (a) and longitudinal (b) OFDI images of the inflated swine lung parenchyma obtained with the TB-OFDI imaging needle. (a) $5.6 \mathrm{~mm} \times 5.6 \mathrm{~mm}$ circumferential cross-sectional OFDI image of lung parenchyma with clear visualization of alveoli. (B) An $8.2 \mathrm{~mm} \times 5.6 \mathrm{~mm}$ YZ longitudinal reconstruction obtained from the location identified in 'a' (yellow dashed line).

To demonstrate the imaging capability of the TB-OFDI imaging probe, we imaged a freshly excised swine lung that was inflated to a physiological pressure of $20 \mathrm{~cm}_{2} \mathrm{O}$. The lung was air filled to closely match the native in vivo conditions. Imaging of the parenchyma was successfully performed at a number of locations chosen throughout the tracheobronchial tree including the very peripheral regions of the lung. Figure 5 shows OFDI images of the lung parenchyma surrounding the needle in logarithmic gray scale. A cross-sectional OFDI image (Fig. 5(a)) and the corresponding longitudinal reslice (Fig. $5(b)$ ) of the volumetric OFDI data (obtained from the position indicated with a yellow dotted line in Fig. 5(a)) demonstrate that the TB-OFDI imaging catheter can clearly resolve alveoli (arrows) within the lung parenchyma.

\section{Discussion and Conclusion}

We have developed a flexible, narrow diameter TB-OFDI catheter that is capable of acquiring high-resolution OFDI images of the peripheral lung. We have successfully demonstrated the feasibility and usability of the TB-OFDI imaging catheter on freshly excised inflated swine lungs. The transbronchial imaging procedure was carried out according to standard clinical bronchoscopy TBNA placement procedures. The experimental results suggest that TB-OFDI may be a useful tool for investigating and potentially increasing the diagnostic yield of peripheral pulmonary lesions. The TB-OFDI catheter presented in this manuscript was designed primarily for pulmonary transbronchial use. The utility of this needle-based design may however extend to other organ systems such as the liver, pancreas, breast, or thyroid.

Although the current catheter sheath is suitably flexible, there is a small risk that it may be damaged or sheared off by the sharp TBNA needle. To mitigate this risk, the inner edge of the needle tip was dulled (Fig. 6). It is not clear at this time if dulling the needle will compromise the ability to aspirate or obtain core biopsy specimens. Future TB-OFDI designs we will investigate the incorporation of rigid hypodermic tubing into the distal portion of the driveshaft to overcome this issue without the need to dull the needle tip.

The current OFDI imaging needle was designed to be side-viewing to provide volumetric assessment of the surrounding tissue. A forward-imaging catheter design may add value by enabling assessment of the tissue prior to positioning the needle within the lesion. This may help to guide needle placement to the target tissue region of interest and to avoid major blood vessels thereby reducing blood contamination in the OFDI images. Forward-imaging catheters have been developed based on the use of coherent fiber bundles [21], paired angled GRIN lenses [22], a GRIN rod [23] and a single-body lensed- 


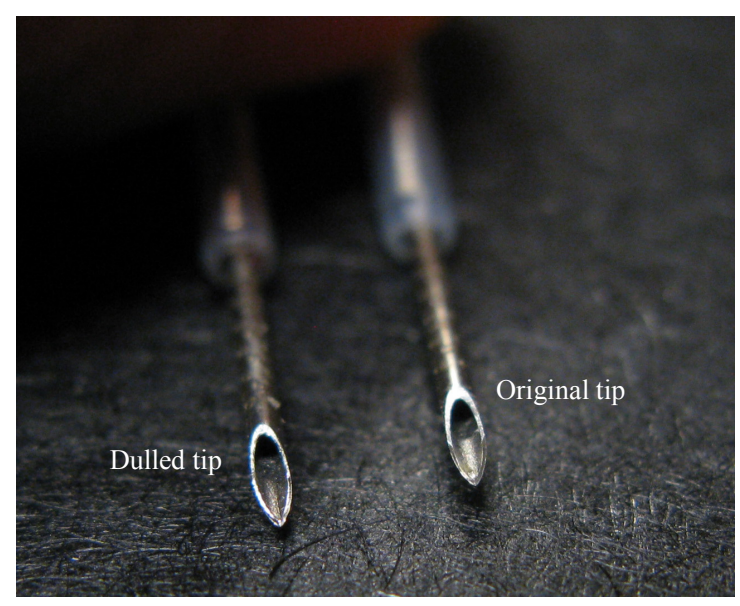

Fig. 6. Photograph of 21-gauge TBNA needle with dulled tip.

fiber design [24], however these catheter designs are difficult to miniaturize while simultaneously providing a sufficiently large imaging field of view.

We believe that the TB-OFDI imaging catheter presented in this manuscript has tremendous potential as a complementary tool to act as a smart needle by confirming the placement of the TBNA needle within a peripheral pulmonary lesion prior to biopsy. We anticipate that the clinical translation of this technology will result in an increase in the diagnostic yield of TBNA of peripheral lesions and may in the future be used to provide real-time optical diagnosis of the lesion. While the TB-OFDI catheter was designed primarily for pulmonary use, the basic design may additionally be useful for other organ systems where FNA or core biopsy procedures are routinely performed.

\section{Acknowledgments}

This work was funded in part by the American Lung Association Biomedical Research Grant (RG-194681-N) and the National Institutes of Health (R00CA134920 and P41EB015903). 